\title{
Prevalence and Correlates of Cannabis Use in Outpatients with Serious Mental Illness Receiving Treatment for Alcohol Use Disorders
}

\author{
Jordan Skalisky, ${ }_{1}^{1-3}$ Emily Leickly, ${ }^{1-3}$ Oladunni Oluwoye, ${ }^{1-3}$ Sterling M. McPherson, ${ }^{2-4}$
}

Debra Srebnik, John M. Roll, ${ }^{2,3}$ Richard K. Ries, ${ }^{5}$ and Michael G. McDonell ${ }^{1-3, *}$

\begin{abstract}
Introduction: People with serious mental illness (SMI) use cannabis more than any other illicit drug. Cannabis use is associated with increased psychotic symptoms and is highly comorbid with alcohol use disorders (AUDs). Despite the national trend toward decriminalization, little is known about the prevalence, correlates, and impact of cannabis use on those with SMI receiving treatment for substance use disorders, a group at high risk for the negative effects of cannabis use.

Methods: In this secondary data analysis, cannabis use prevalence, correlates, and impact on treatment outcomes were examined in 121 adults with cooccurring SMI and AUDs receiving outpatient addiction treatment in a randomized trial of contingency management (CM) for alcohol. Prevalence and frequency of cannabis use were calculated across the 7-month study period using self-report and urine tests. Cannabis users were compared with nonusers by SMI diagnosis, psychiatric symptoms, medical problems, legal problems, and HIV-risk behavior. The relationship between cannabis use and longest duration of alcohol abstinence in participants randomized to CM $(n=40)$ was assessed.

Results: Fifty-seven (47\%) of participants submitted at least one cannabis-positive urine sample during the study. Out of the 2834 total samples submitted, 751 (27\%) were positive for cannabis. Cannabis users were 2.2 times more likely to submit an alcohol-positive sample, and 2.5 times more likely to submit a cocaine-positive sample at baseline, relative to noncannabis users $(p=0.01)$. Cannabis users were more likely to engage in risky sexual behavior $(p=0.01)$ and to report being homeless ( $p=0.03$ ) than nonusers. When controlling for pretreatment alcohol use, the relationship between comorbid cannabis use and alcohol abstinence during CM was not significant $(p=0.77)$.

Conclusion: Rates of comorbid cannabis use were high in this sample of adults with SMI and AUDs. Cannabis use was correlated with recent alcohol and cocaine use, risky sexual behavior, and homelessness, but not with alcohol abstinence during CM.
\end{abstract}

Keywords: alcohol use disorders; cannabis; contingency management; serious mental illness

\footnotetext{
${ }^{1}$ Initiative for Research and Education to Advance Community Health, Washington State University, Spokane, Washington.

${ }^{2}$ Elson S. Floyd College of Medicine, Washington State University, Spokane, Washington.

${ }^{3}$ Program for Excellence in Addiction Research, Washington State University, Spokane, Washington.

${ }^{4}$ Providence Medical Research Center, Providence Health Care, Spokane, Washington.

${ }^{5}$ Department of Psychiatry and Behavioral Sciences, University of Washington School of Medicine, Seattle, Washington.
}

*Address correspondence to: Michael G. McDonell, PhD, Initiative for Research and Education to Advance Community Health, Washington State University, College of Medicine, PO Box 1495, Spokane, WA 99210-1495, E-mail: mmcdonell@wsu.edu

(C) Jordan Skalisky et al. 2017; Published by Mary Ann Liebert, Inc. This is an Open Access article distributed under the terms of the Creative Commons Attribution License, which permits unrestricted use, distribution, and reproduction in any medium, provided the original work is properly cited. 


\section{Introduction}

Alcohol and cannabis are among the most commonly used substances worldwide. ${ }^{1}$ Within the United States, 136 million adults reported alcohol use and 20.5 million reported cannabis use in the past 30 days. ${ }^{2}$ Cannabis use and alcohol use disorders (AUDs) are highly comorbid. Cannabis users are 32\% more likely than nonusers to have persistent AUDs. ${ }^{3}$ In the National Epidemiologic Survey on Alcohol and Related Conditions, $40 \%$ of adults with AUDs reported cannabis use in the past 12 months. ${ }^{4}$ Co-occurring cannabis and alcohol use is associated with increased aggressive behavior, interactions with the criminal justice system, and physical health problems. ${ }^{5}$ Studies investigating the impact of comorbid cannabis use on the treatment of alcohol and other drugs have been inconclusive. ${ }^{6-8}$

Adults with serious mental illness (SMI), defined as recurring major depressive, bipolar, and schizophrenia spectrum disorders, have a lifetime prevalence of cannabis use twice as high as that of the general population..$^{9-11}$ Unfortunately, nearly $50 \%$ of adults with SMI will be diagnosed with an AUD during their lifetime. ${ }^{12}$ Adults with SMI and AUDs may be at a greater risk of negative consequences of co-occurring cannabis and alcohol use. Cannabis use is associated with more persistent psychotic symptoms, such as increased hallucinations, and alcohol use is associated with increased risk of HIV infection, homelessness, incarceration, and intentional and unintentional self-harm. ${ }^{1,9,13,14}$

Less is known about the prevalence and impact of comorbid cannabis and alcohol use in adults with SMI. One study of youth at high risk for psychosis found that although cannabis use disorders were associated with increased risk of developing psychosis, alcohol use moderated this relationship, suggesting that assessing comorbid alcohol and cannabis use may be important when understanding the relationship between substance use and the onset of psychosis. ${ }^{15}$ Given the high prevalence of cannabis and alcohol use in adults with SMI, as well as previous inconclusive findings regarding the impact of comorbid cannabis use on alcohol and other drug treatment outcomes among populations without SMI, research is needed to better understand the frequency, severity, correlates, and impact of cannabis use on outcomes in adults with SMI.

Understanding the prevalence, correlates, and relationship of cannabis use with alcohol use and illicit substances in individuals with SMI is of increasing importance. As of April 2017, 29 states and the District of Columbia have legalized medical use of cannabis. Therefore, the objec- tives of this study were to determine the prevalence and frequency of cannabis use, demographic and clinical correlates of cannabis use, and the relationship between cannabis use and alcohol treatment outcomes in adults with AUDs and SMI.

\section{Materials and Methods}

Setting

Participants were recruited between 2012 and 2015 from community health clinics in King County, Washington, that provide both mental health and addiction treatment. It should be noted that Washington State's Initiative 502 decriminalizing recreational cannabis use was passed in December 2012 and the first recreational cannabis was sold in the state on July 8, 2014.

\section{Participants}

Participants were recruited as part of a larger study of the impact of contingency management (CM) on adults with SMI and AUDs. Participants met criteria for (1) Diagnostic and Statistical Manual of Mental Disorders, fourth edition (text revision) (DSM-IV-TR) diagnoses of alcohol dependence, (2) recurring major depressive disorder, bipolar disorder I or II, schizophrenia, or schizoaffective disorder, as assessed by the Mini-International Neuropsychiatric Interview (MINI), ${ }^{16}$ and (3) alcohol use in at least 5 out of 30 days before baseline. Participants were 18-65 years of age and enrolled in treatment as usual for alcohol dependence. Exclusion criteria included significant risk of dangerous alcohol withdrawal, DSM-IV-TR diagnosis of current (within the past year) drug dependence that suggested physiological dependence (i.e., withdrawal and tolerance), any medical or psychiatric conditions that could compromise safe study participation, or inability to provide informed consent. The University of Washington's Institutional Review Board approved study procedures.

\section{Measures}

Demographic information was collected at the baseline interview. Participants completed the MINI to assess for alcohol, drug, mood, and psychotic disorders. At baseline and weeks 4, 8, 12, 16, 20,24, and 28, the Addiction Severity Index-Lite was used to assess for days of alcohol use, drinking to intoxication, and cannabis and other drug use in the previous 30 days. ${ }^{17}$ Psychiatric symptom severity was assessed using the Positive and Negative Syndrome Scale (PANSS), a clinician-rated measure of schizophrenia-spectrum symptoms, and the Brief Symptom Inventory (BSI), a self-report measure of 
general psychopathology. The HIV Risk Taking Behavior Scale (HRBS) was used to assess risky sexual behavior and HIV risk. ${ }^{18-20}$ Participants received gift cards to a local grocery store for baseline and follow-up interviews.

Participants submitted urine samples for alcohol and drug testing three times a week during the 4week prerandomization phase and the 12 -week treatment period. The primary alcohol use outcome was Diagnostic Reagents Incorporated ethyl glucuronide enzyme immunoassay (EtG-I) conducted onsite using a Thermo Fisher Indiko analyzer (Fremont, CA). Tests were conducted using EtG $100 \mathrm{ng} / \mathrm{mL}, 500 \mathrm{ng} / \mathrm{mL}, 1000 \mathrm{ng} / \mathrm{mL}, 2000 \mathrm{ng} /$ $\mathrm{mL}$, and Negative calibrators and EtG $100 \mathrm{ng} / \mathrm{mL}$ and $375 \mathrm{ng} / \mathrm{mL}$ controls. Calibrations occurred weekly and samples were analyzed on the day of collection. Pretreatment EtG levels were calculated for each participant that ranged from 0 to $2000 \mathrm{ng} / \mathrm{mL}$. Pretreatment heavy drinking was defined as an average pretreatment EtG level of $>499 \mathrm{ng} / \mathrm{mL}$, a cutoff equivalent to regular heavy drinking, compared with $<500 \mathrm{ng} / \mathrm{mL}$ (light drinking). ${ }^{21}$ During the treatment phase, samples were considered alcohol negative if EtG $<150 \mathrm{ng} / \mathrm{mL}$. Longest duration of alcohol abstinence (LDA) was defined as the highest consecutive number of EtG-negative urine tests (range 0-36 visits) during treatment. Point-of-care urine drug tests (Integrated E-Z Split Key Cup II; Innovacon, Inc., San Diego, CA) were used to assess cannabis, methamphetamines, amphetamines, opioids, and cocaine use. The cutoff for cannabis was $50 \mathrm{ng} / \mathrm{mL}$.

\section{Intervention}

The study consisted of a baseline interview, a 4-week pretreatment phase (weeks 1-4), a 12-week treatment phase (weeks 5-12), and a 3-month follow-up phase (weeks 13-28). The variable magnitude of reinforcement procedure was used to reinforce submission of urine samples during the 4-week prerandomization phase and during the treatment period. ${ }^{22}$ Participants were asked to attend study visits three times a week on Mondays, Wednesdays, and Fridays. All participants participated in a 4-week prerandomization phase wherein they were eligible for three prize draws each time they submitted a urine sample, regardless of EtG results. After completing the prerandomization phase, participants continued to receive treatment as usual and were randomized into two groups, noncontingent control (NC) or CM. NC participants were eligible for incentives despite the results of their urine tests for alcohol. Participants in the CM condition received prize draws only when their urine test was negative for alcohol (EtG $<150 \mathrm{ng} / \mathrm{mL}$ ). During the CM treatment phase, par- ticipants also received a $\$ 5$ or $\$ 10$ gift card for attending some or all of their scheduled outpatient addiction treatment groups each week. NC participants received gift cards equal to those earned by the $\mathrm{CM}$ group during the previous week, regardless of whether they attended addiction treatment group sessions.

\section{Analyses}

Descriptive analyses were conducted to describe the number of individuals who submitted a cannabis-positive urine test or reported cannabis use during the study. The total number of cannabis-positive urine samples was calculated across the entire sample $(N=121)$ and the mean number of cannabis-positive urine tests was calculated among cannabis users. Cannabis users were defined as participants who submitted at least one cannabis-positive urine sample throughout the study. Differences were explored between cannabis users and noncannabis users using chi-square tests for categorical variables, including sex, race, SMI diagnosis, and urine drug (methamphetamines, amphetamines, opioids, and cocaine) and alcohol use. Independent samples $t$-tests were used for continuous variables, such as HRBS, BSI, and PANSS scores. Analysis of covariance (ANCOVA) was used to determine whether the main effect of cannabis use was associated with the LDA from alcohol in the randomized sample accounting for treatment group membership (CM vs. control condition) and pretreatment levels of EtG-assessed heavy drinking (EtG $>499 \mathrm{ng} / \mathrm{mL}$ ). We also conducted an ANCOVA in the CM group only $(n=40)$ to determine whether the main effect of cannabis use was associated with the LDA, accounting for pretreatment levels of EtG-assessed heavy drinking (EtG $>499 \mathrm{ng} / \mathrm{mL}$ ). The criterion for statistical significance was set at alpha $p<0.05$ and analyses were conducted using IBM SPSS version 24 .

\section{Results}

A total of 121 individuals met inclusion criteria and provided informed consent. Participants had a mean age of 45.4 (standard deviation $[\mathrm{SD}]=10.1$ ), 34.7\% $(n=42)$ were female, and $47 \%(n=57)$ were nonwhite. Thirty-five percent $(n=42)$ were diagnosed with major depressive disorder, $38 \%(n=46)$ with bipolar I or II disorders, and $27 \%(n=33)$ with schizophrenia spectrum disorders. Baseline demographics and clinical characteristics are reported in Table 1.

A total of 2834 urine samples were submitted throughout the study, with participants each submitting a mean of $23.4(\mathrm{SD}=16.2)$ urine samples during 
Table 1. Baseline Demographic and Clinical Characteristics of the Evaluative Sample $(N=121)$

\begin{tabular}{|c|c|c|c|c|}
\hline & \multicolumn{2}{|c|}{ Cannabis users } & \multicolumn{2}{|c|}{ Noncannabis users } \\
\hline & \multicolumn{2}{|c|}{$n=57$} & \multicolumn{2}{|c|}{$n=64$} \\
\hline & $n(\%)$ & Mean (SD) & $n(\%)$ & Mean (SD) \\
\hline \multicolumn{5}{|l|}{ Demographic characteristics } \\
\hline Age & 57 & $43.7(10.9)$ & 64 & $46.9(9.2)$ \\
\hline Male & $39(68.4)$ & & $40(62.5)$ & \\
\hline \multicolumn{5}{|l|}{ Race } \\
\hline Caucasian & $34(59.6)$ & & $30(46.9)$ & \\
\hline African American & $11(19.3)$ & & $23(35.9)$ & \\
\hline Other & $12(21.1)$ & & $11(17.2)$ & \\
\hline Homeless & $22\left(18.2^{\mathrm{a}}\right)$ & & $13\left(10.7^{\mathrm{a}}\right)$ & \\
\hline \multicolumn{5}{|l|}{ Diagnosis } \\
\hline Major depressive disorder & 18 (31.6) & & $24(37.5)$ & \\
\hline Bipolar disorder & $24(42.1)$ & & $22(34.4)$ & \\
\hline Schizophrenia-spectrum disorder & $15(26.3)$ & & $18(25.1)$ & \\
\hline \multicolumn{5}{|l|}{ HRBS } \\
\hline Injection drug use & $4(7.1)$ & & $9(14.3)$ & \\
\hline Sexual behavior & 56 & $3.5^{\mathrm{a}}(4.1)$ & 63 & $1.8^{\mathrm{a}}(2.5)$ \\
\hline \multicolumn{5}{|l|}{ BSI } \\
\hline Total & 54 & $90.2(47.9)$ & 62 & $78.5(38.4)$ \\
\hline \multicolumn{5}{|l|}{ PANSS } \\
\hline Positive symptoms & 55 & $12.0(4.2)$ & 63 & $12.2(4.4)$ \\
\hline Negative symptoms & 55 & $13.2(5.1)$ & 63 & $13.6(5.4)$ \\
\hline Emotional distress & 55 & $17.6(4.8)$ & 63 & $17.9(4.0)$ \\
\hline Disorganization & 55 & $20.1(5.4)$ & 63 & $20.4(5.4)$ \\
\hline Excitement & 55 & $13.5(14.0)$ & 63 & $14.0(3.6)$ \\
\hline \multicolumn{5}{|l|}{ Other drug positives } \\
\hline Alcohol & $36\left(63.2^{\mathrm{a}}\right)$ & & $18\left(28.1^{\mathrm{a}}\right)$ & \\
\hline Amphetamines & $5(8.8)$ & & $3(4.7)$ & \\
\hline Methamphetamines & $4(7.0)$ & & $3(4.7)$ & \\
\hline Cocaine & $18\left(31.6^{\mathrm{a}}\right)$ & & $8\left(12.5^{\mathrm{a}}\right)$ & \\
\hline Opiates & $2(3.5)$ & & $2(3.1)$ & \\
\hline
\end{tabular}

${ }^{\mathrm{a}}$ Difference is significant at $p<0.05$.

BSI, Brief Symptom Inventory; HRBS, HIV Risk Taking Behavior Scale; PANSS, Positive and Negative Syndrome Scale; SD, standard deviation.

the study. Among the entire sample, 11.6\% $(n=14)$ reported having a medical marijuana card. However, among those who were cannabis users $(n=57)$ during the study, only $11(19.3 \%)$ reported having a medical marijuana card. Across the duration of the entire study, 751 (26.5\%) cannabis-positive urine samples were submitted and $47 \%(n=57)$ of participants submitted at least one cannabis-positive urine sample. Participants who submitted at least one cannabis-positive sample had a mean of $13.2(\mathrm{SD}=13.5)$ cannabis-positive samples. Although 57 participants submitted cannabispositive urine samples throughout the study, only 52 self-reported cannabis use in the 30 days before baseline. Among the 52 participants who reported cannabis use in the 30 days before baseline, participants reported a mean 11.7 days of cannabis use $(\mathrm{SD}=10.9)$, with $11.5 \%(n=6)$ reporting cannabis use every day.

As described in Table 1, cannabis users were more likely to submit an alcohol-positive urine test at baseline $(n=36$,
$63.2 \%)$, relative to noncannabis users $(n=18,28.1 \%)$, $\chi^{2}(1)=15, p<0.001$. Cannabis users also had higher mean HIV-risk scores on the sexual behavior subscale of the HRBS (mean $=3.5, \mathrm{SD}=4.1$ ), relative to noncannabis users (mean $=1.8, \mathrm{SD}=2.5) t(89.9)=-2.74, p=0.01$. Cannabis users were more likely to submit a cocaine-positive urine sample, $\chi^{2}(1)=6.5, p=0.01$ and be homeless, $\chi^{2}(1)=4.9, p=0.03$, relative to noncannabis users. Medical and legal problems were not significantly different across cannabis and noncannabis users.

In terms of the impact of cannabis use on treatment outcomes in the randomized sample, pretreatment heavy drinking, $\mathrm{F}(1,71)=4.99, p<0.05$, but not cannabis use, $\mathrm{F}(1,71)=0.45, p=0.51$, or treatment group, $\mathrm{F}(1,71)=2.43, p=0.12$, was significantly associated with LDA from alcohol during CM, with heavy drinkers having a shorter duration of abstinence relative to those who did not engage in heavy drinking. This finding was replicated within the CM group $(n=40)$. Pretreatment 
heavy drinking, $\mathrm{F}(1,37)=7.45, p<0.05$, but not cannabis use, $\mathrm{F}(1,37)=0.09, p=0.77$, was significantly associated with LDA from alcohol during CM.

\section{Discussion}

Approximately half the participants diagnosed with alcohol dependence and SMI participating in a clinical trial of CM for AUDs submitted at least one cannabispositive urine sample during the study. More than one out of every four urine samples submitted by cannabis users was cannabis positive, suggesting regular ongoing use. Cannabis use was not associated with duration of alcohol abstinence. This is consistent with findings that pretreatment cannabis use was not associated with alcohol abstinence, regardless of whether it was for medical or recreational purposes. ${ }^{7}$ This provides important information to clinicians who must decide whether they should treat comorbid cannabis use in their patients receiving treatment for other substance use disorders, as it may not affect the primary substance use treatment outcome.

Cannabis users engaged in higher levels of sexual HIV-risk behaviors and were more likely to have an alcohol or cocaine-positive urine sample at baseline, indicating higher levels of very recent alcohol and drug use. Cannabis users were also more likely to report homelessness. The combination of AUDs and cannabis among adults with SMI may be associated with increased severity of substance use and risky behaviors that may, in turn, be related to an increased likelihood of homelessness. These correlations may suggest that cannabis users have a higher level of substance use severity or that cannabis, alcohol, and cocaine use, and HIV-risk behaviors are all associated with an underlying factor, such as high levels of impulsivity. Further research is needed to better understand the nature of these associations.

There are several limitations to these findings. Foremost, the research was not an epidemiological investigation as we only recruited individuals with SMI enrolled in an alcohol treatment study. Therefore, results might not generalize to the entire population of adults with SMI, or to adults with SMI who also use illicit drugs. We were also not able to describe the exact amount, type, and route of administration (e.g., smoked, eaten, and vaped) of daily cannabis use. In addition, our subsample of individuals randomized to CM was relatively small $(n=40)$.

Despite the limitations of this secondary data analysis, results highlight the high prevalence of cannabis use among SMI outpatients receiving AUD treatment and provide initial evidence that comorbid cannabis use does not affect CM outcomes. Although the public health debate regarding the health costs and benefits of cannabis legalization has focused on youth, including youth at risk for SMI, less is known about the impact of cannabis use on other at-risk populations such as adults with SMI. As an already vulnerable population, it is critical to understand how cannabis use may exacerbate the many problems adults with SMI and AUDs already face. In addition, it is unclear when adults with SMI and AUDs are engaging in cannabis use and how this affects HIVrisk behaviors and homelessness. For example, we do not know whether these outcomes are largely driven by the SMI, AUD, or cannabis use, or a combination of psychopathology and substance use. Larger epidemiological studies are needed to more definitively understand the prevalence and correlates of cannabis use and its impact on psychiatric and substance abuse treatment outcomes.

\section{Acknowledgment}

The National Institute of Alcohol Abuse and Alcoholism funded this research (R01 AA020248-1A1, PI: McDonell).

\section{Author Disclosure Statement}

Drs. McPherson and Roll have received research funding from the Bristol-Myers Squibb Foundation. This funding is in no way related to the investigation reported here. Dr. Ries has been on the speaker bureaus of Janssen, Alkermes, and Reckitt Benckiser in the past three years but has no disclosures to report. All other authors have no disclosures to report.

\section{References}

1. World Health Organization. Global status report on alcohol and health. World Health Organization: Geneva, Switzerland, 2014:1-100.

2. Center for Behavioral Health Statistics and Quality. Key substance use and mental health indicators in the United States: results from the 2015 national survey on drug use and health. HHS Publication: Rockville, MD, 2016.

3. Weinberger AH, Platt J, Goodwin RD. Is cannabis use associated with an increased risk of onset and persistence of alcohol use disorders? A threeyear prospective study among adults in the United States. Drug Alcohol Depend. 2016;161:363-367.

4. Blanco C, Hasin DS, Wall MM, et al. Cannabis use and risk of psychiatric disorders. JAMA Psychiatry. 2016;73:388-395.

5. Subbaraman MS, Kerr WC. Simultaneous versus concurrent use of alcohol and cannabis in the national alcohol survey. Alcohol Clin Exp Res. 2015;39:872-879.

6. Aharonovich E, Garawi F, Bisaga A, et al. Concurrent cannabis use during treatment for comorbid ADHD and cocaine dependence: effects on outcome. Am J Drug Alcohol Abuse. 2006;32:629-635.

7. Alessi SM, Rash C, Petry NM. Contingency management is efficacious and improves outcomes in cocaine patients with pretreatment marijuana use. Drug Alcohol Depend. 2011;118:62-67.

8. Mayet $A$, Lions $C$, Roux $P$, et al. Variations in cannabis use level and correlates in opiate-users on methadone maintenance treatment: a French prospective study. J Subs Abuse Treat. 2015;58:100-105.

9. Bruins J, Pijnenborg MG, Bartels-Velthuis AA, et al. Cannabis use in people with severe mental illness: the association with physical and mental health-a cohort study. A pharmacotherapy monitoring and outcome survey study. J Psychopharmacol. 2016;30:354-362. 
10. Foti DJ, Kotov R, Guey LT, et al. Cannabis use and the course of schizophrenia: 10-year follow-up after first hospitalization. Am J Psychiatry. 2010;167:987-993.

11. Green B, Young R, Kavanagh D. Cannabis use and misuse prevalence among people with psychosis. Br J Psychiatry. 2005;187:306-313.

12. Regier DA, Farmer ME, Rae DS, et al. Comorbidity of mental disorders with alcohol and other drug abuse. Results from epidemiologic catchment area (ECA) study. JAMA. 1990;264:2511-2518.

13. Henquet $C$, van Os J, Kuepper R, et al. Psychosis reactivity to cannabis use in daily life: an experience sampling study. Br J Psychiatry. 2010;196: 447-453.

14. Substance Abuse and Mental Health Services Administration. Substance abuse treatment for persons with co-occurring disorders, treatment improvement protocol (TIP) series, no. 42. HHS Publication: Rockville, MD, 2005.

15. Auther AM, Cadenhead KS, Carrion RE, et al. Alcohol confounds relationship between cannabis misuse and psychosis conversion in a highrisk sample. Acta Psychiatr Scand. 2015;132:60-68.

16. Sheehan DV, Lecruibier $Y$, Sheehan KH, et al. The mini-international neuropsychiatric interview (M.I.N.I.): the development and validation of a structured diagnostic psychiatric interview for DSMM-IV and ICD-10. J Clin Psychiatry. 1998;59:22-57.

17. Cacciola JS, Alterman AI, McLellan AT, et al. Initial evidence for the reliability and validity of a "lite" version of the addiction severity index. Drug Alcohol Depend. 2007;87:297-302.

18. Kay SR, Flszbein A, Opfer LA. The positive and negative syndrome scale (PANSS) for schizophrenia. Schizophr Bull. 1987;13: 261.

19. Preston NJ, Harrison TJ. The brief symptom inventory and the positive and negative syndrome scale: discriminate validity between a selfreported and observational measure of psychopathology. Compr Psychiatry. 2003:44:220-226.

20. Ward J, Darke S, Hall W. The HIV risk-taking behaviour scale (HRBS) manual. National Drug and Alcohol Research Centre: University of New South Wales, Sydney, 1990.
21. McDonell MG, Skalisky J, Leickly $\mathrm{E}$, et al. Using ethyl glucuronide in urine to detect light and heavy drinking in alcohol dependent outpatients. Drug Alcohol Depend. 2015;157:184-187.

22. McDonell MG, Leickly E, McPherson S, et al. A randomized controlled trial of ethyl glucuronide-based contingency management for outpatients with co-occurring alcohol use disorders and serious mental illness. Am J Psychiatry. 2017;174:370-377.

Cite this article as: Skalisky J, Leickly E, Oluwoye O, McPherson SM, Srebnik D, Roll JM, Ries RK, McDonell MG (2017) Prevalence and correlates of cannabis use in outpatients with serious mental illness receiving treatment for alcohol use disorders, Cannabis and Cannabinoid Research 2:1, 133-138, DOI: 10.1089/can.2017.0006.

$\begin{aligned} & \text { Abbreviations Used } \\ & \text { ANCOVA }=\text { analysis of covariance } \\ & \mathrm{AUDs}=\text { alcohol use disorders } \\ & \mathrm{BSI}=\text { Brief Symptom Inventory } \\ & \mathrm{CM}=\text { contingency management } \\ & \mathrm{EtG}-\mathrm{I}=\text { ethyl glucuronide enzyme immunoassay } \\ & \mathrm{HRBS}=\text { HIV Risk Taking Behavior Scale } \\ & \mathrm{LDA}=\text { longest duration of alcohol abstinence } \\ & \mathrm{MINI}=\text { Mini-International Neuropsychiatric Interview } \\ & \mathrm{NC}=\text { noncontingent control } \\ & \mathrm{PANSS}=\text { Positive and Negative Syndrome Scale } \\ & \mathrm{SD}=\text { standard deviation } \\ & \mathrm{SMI}=\text { serious mental illness }\end{aligned}$

Cannabis and

Cannabinoid

Research
- Immediate, unrestricted online access

- Rigorous peer review

- Compliance with open access mandates

- Authors retain copyright

- Highly indexed

- Targeted email marketing 